\title{
Lithium alters brain activation in bipolar disorder in a task- and state-dependent manner: an fMRI study
} Peter H Silverstone*1, Emily C Bell ${ }^{1}$, Morgan C Willson ${ }^{2}$, Sanjay Dave ${ }^{1}$ and Alan H Wilman ${ }^{2}$

Address: ${ }^{1}$ Department of Psychiatry, Faculty of Medicine, University of Alberta, 1E1.07 MacKenzie Center, 8440-112 Street, Edmonton, Alberta, T6G 2B7, Canada and 2Department of Biomedical Engineering, Faculty of Medicine, University of Alberta, 1071 Research Transition Facility, 8308 114 Street, Edmonton, Alberta, T6G 2V2, Canada

Email: Peter H Silverstone* - peter.silverstone@ualberta.ca; Emily C Bell - ebell@ualberta.ca;

Morgan C Willson - morgan.willson@med.ucalgary.ca; Sanjay Dave - sanjay.dave@ualberta.ca; Alan H Wilman - alan.wilman@ualberta.ca

* Corresponding author

Published: 19 July 2005

Annals of General Psychiatry 2005, 4:14 doi:10.1186/1744-859X-4-14

This article is available from: http://www.annals-general-psychiatry.com/content/4/I/14

(c) 2005 Silverstone et al; licensee BioMed Central Ltd.

This is an Open Access article distributed under the terms of the Creative Commons Attribution License (http://creativecommons.org/licenses/by/2.0), which permits unrestricted use, distribution, and reproduction in any medium, provided the original work is properly cited.

\begin{abstract}
Background: It is unknown if medications used to treat bipolar disorder have effects on brain activation, and whether or not any such changes are mood-independent.

Methods: Patients with bipolar disorder who were depressed $(n=5)$ or euthymic $(n=5)$ were examined using fMRI before, and 14 days after, being started on lithium (as monotherapy in 6 of these patients). Patients were examined using a word generation task and verbal memory task, both of which have been shown to be sensitive to change in previous fMRI studies. Differences in blood oxygenated level dependent (BOLD) magnitude between the pre- and post-lithium results were determined in previously defined regions of interest. Severity of mood was determined by the Hamilton Depression Scale for Depression (HAM-D) and the Young mania rating scale (YMRS).

Results: The mean HAM-D score at baseline in the depressed group was $15.4 \pm 0.7$, and after 2 weeks of lithium it was $11.0 \pm 2.6$. In the euthymic group it was $7.6 \pm 1.4$ and $3.2 \pm 1.3$ respectively. At baseline mean BOLD signal magnitude in the regions of interest for the euthymic and depressed patients were similar in both the word generation task ( $1.56 \pm 0.10$ and $1.49 \pm 0.10$ respectively) and working memory task $(1.02 \pm 0.04$ and $\mathrm{I} .12 \pm 0.06$ respectively). However, after lithium the mean BOLD signal decreased significantly in the euthymic group in the word generation task only $(1.56 \pm 0.10$ to $I .00 \pm 0.07, p<0.00 \mathrm{I})$. Post-hoc analysis showed that these differences were statistically significant in Broca's area, the left precentral gyrus, and the supplemental motor area.

Conclusion: This is the first study to examine the effects of lithium on brain activation in bipolar patients. The results suggest that lithium has an effect on euthymic patients very similar to that seen in healthy volunteers. The same effects are not seen in depressed bipolar patients, although it is uncertain if this lack of change is linked to the lack of major improvements in mood in this group of patients. In conclusion, this study suggests that lithium may have effects on brain activation that are task- and state-dependent. Given the small study size and the mildness of the patient's depression these results require replication.
\end{abstract}




\section{Background}

Studies suggest that there are trait abnormalities in cognitive functioning in bipolar disorder, i.e. cognitive changes which are seen when patients are either manic or depressed but which are also seen when patients are euthymic. These are seen in studies of sustained attention, executive functioning, and verbal learning $[1,2]$. The evidence to date suggests that there are trait cognitive abnormalities in bipolar patients, although it is possible these can be exacerbated by medication and the duration of illness [3]. However, it should be recognized that some of the treatments for bipolar disorder, particularly lithium, can also cause cognitive deficits particularly in tests of verbal memory $[4,5]$.

It remains uncertain how these cognitive deficits may be reflected in functional changes in brain activation. One study comparing depressed and euthymic bipolar patients found regional cerebral blood flow (rCBF) changes in both groups in multiple brain regions [6]. Another study utilizing fMRI examined patients who were depressed, manic, and euthymic and suggested there was a trait abnormality in the prefrontal cortex [7]. An fMRI study comparing euthymic patients to controls also suggested changes in limbic and visual cortex may represent trait abnormalities of brain functioning [8]. Another fMRI study in euthymic bipolar patients found significantly increased activation compared to controls in a working memory task in several different brain regions [9]. Thus, studies to date support suggestions of underlying functional changes in bipolar patients that to some extent may reflect the well recognized cognitive deficits.

It is of considerable interest to determine whether functional changes seen in bipolar patients may be attenuated by treatment, but to date there have been no studies examining the possible effects of treatment upon brain activation. Lithium remains one of the primary treatments for bipolar disorder [10], and as noted also can have independent cognitive effects. In a previous study we have shown that in healthy volunteers treatment with lithium for 2 weeks led to a decrease in mean BOLD magnitude in a word generation task (unpublished information). However, one of the problems of examining patients before and after treatment is that if the mood changes following medication, it can be difficult to know what the effects of medication alone are. For these reasons the present study examines both bipolar patients who are depressed and those who are euthymic. Given previous findings about cognitive deficiencies, the two cognitive tests used in the present study were firstly one that focused on sustained attention (working memory task) and the second was a verbal task (word generation task).

\section{Materials and methods Subjects}

The study was approved by the ethics committee at the University of Alberta and all subjects gave full informed consent. Bipolar patients aged 18 - 65 who were right handed were eligible and were recruited from the Bipolar Clinic at the University of Alberta and by referral from colleagues. A full personal and family history was obtained and diagnosis was made using Diagnostic and Statistical Manual of Mental Disorders version IV (DSM-IV) criteria for bipolar disorder [11], using a the structured clinical interview for DSM-IV (SCID). All patients also had a physical examination including weight and blood pressure, biochemical screening including full blood count, urea and electrolytes, and measurement of liver and renal functioning. Patients who had any another Axis I diagnosis (including an anxiety disorder or drug or alcohol dependence or abuse), or who had clinically significant medical findings, were excluded from the study. Depressed symptomatology was determined by clinical interview using DSM-IV criteria. Symptom severity for depression was determined using the 17-item Hamilton Depression rating scale (HAM-D) [12], and the presence of manic symptoms was measured using the Young Mania Rating Scale (YMRS) [13]. Patients were determined as euthymic if they had been clinically stable for at least 3 months and had a HAM-D score of less than 12 and a YMRS of less than 6 .

\section{Cognitive Tasks and Study Design Working Memory Task}

The working memory task consisted of two conditions. In the first condition there was a series of ten arrows pointing to the left or to the right. Subjects responded by pressing the left or right button. In the second condition a five-digit number was displayed for four seconds with the instruction 'Remember this Number'. Immediately after, a series of ten random single digits was presented. Subjects were asked to respond by pressing one button for yes and one for no if the single digit was or was not in the memorized number. In all, each condition was presented seven times in twenty-four second blocks pseudo-randomly arranged [14].

\section{Word Generation Task}

The word generation task had two conditions. In the first condition subjects were asked to repeat the word 'REST' silently until another instruction was given. In the second condition a series of ten single letters randomly chosen from the alphabet were displayed at 4-second intervals. During the display of a single letter, subjects responded by thinking of as many words as possible that begin with that letter and repeating them silently until another letter is presented or the 'REST' instruction appears. The 
experiment consisted of five forty second blocks of each condition beginning with 'REST' [14].

\section{Functional magnetic resonance imaging (fMRI) study design}

All imaging parameters and tasks were identical to those described previously [14]. The fMRI images were collected on a 1.5T Siemens Sonata scanner, using a single shot EPI gradient echo sequence $(\mathrm{TR}=4010 \mathrm{~ms}, \mathrm{TE}=50 \mathrm{~ms}, 1.7 \times$ $1.7 \mathrm{~mm}, 4 \mathrm{~mm}$ thick) to acquire 30 contiguous slices obtained at an oblique angle along the AC-PC line. A high resolution T1 weighted MPRAGE sequence was also obtained for overlay of the functional analysis.

\section{fMRI Data Analysis}

Pre-processing and analysis was performed using Statistical Parametric Mapping (SPM), 1999 version [15]. All functional images were realigned during pre-processing to accommodate and correct for any head motion. Realignment was performed using a 6-parameter rigid body transformation and a mean image was created of the entire time series for each data set. Sessions with realignment parameters of greater than $4 \mathrm{~mm}$ in the direction of translation (along the $\mathrm{x}, \mathrm{y}, \mathrm{z}$ axis) were excluded from the final statistical analysis, as were sessions with motion greater than 0.05 radians in a rotational plane (pitch, roll, yaw). The mean image was then spatially normalized to the MNI template brain using a 12-parameter affine transformation with 12 non-linear iterations and $7 \times 8 \times 7$ basis functions. The spatial transformations derived from normalizing the mean image to the template were then applied to the T2* weighted EPI functional images. After normalization, all volumes were resampled to $2 \times 2 \times 2$ $\mathrm{mm}$ voxels using tri-linear interpolation in space. Finally, all functional images were smoothed with an 8-mm full width at half-maximum isotropic Gaussian kernel to compensate for between subject variability and allow Gaussian random field theory to give corrected statistical inferences [16]. Initial analysis was performed separately for each subject for each task. The model specified for each task was kept identical for all subjects and sessions to create identical design matrices. As part of this analysis three more pre-processing steps were performed using SPM99. First the data was high passed filtered to remove low-frequency drifts in the signal. In addition, the data was low pass filtered using the hemodynamic response function to remove high frequency noise. Effects due to global intensity fluctuations were removed when the data was proportionally scaled to a global mean of 100 . The time series for each data set was analyzed according to the general linear model. Previously, we have performed a group analysis on 18 volunteers [14] by constructing a fixed effects model for each task. Regions of interest for each task were then compiled using the most significantly activated voxels from the group average generation maps and thus pre- determined regions of interested were examined. Region of interest (ROI) images were constructed using automated anatomical labeling software [17] running with MRIcro software [18]. In the present study we assessed activation in these previously identified ROIs.

For each subject the individual activation maps generated during single-subject analysis were used to identify the change in BOLD signal magnitude [14]. The BOLD signal intensity change was calculated based on regions of interest using the MARSBAR toolbox for SPM [19] over a sevenvoxel sphere centered on the most significantly active voxel in each ROI. The fitted response (or BOLD signal intensity change) is expressed in percentage of whole brain mean. Because the global brain mean in the voxel-wise analysis was scaled to 100 , this signal change represents the percentage of signal change with respect to the global mean intensity of the scaled images. The number of voxels over which the fitted response was calculated was kept small in order to minimize averaging over non-significant voxels or large veins [20]. Subsequently statistical calculations for BOLD signal magnitude were based on the average response calculated from the plateau portion of the hemodynamic response (eight seconds after stimulus origination until stimulus termination.

\section{Statistical analysis}

The mean BOLD signal magnitude in each task was calculated over all the ROIs (for that task) and an analysis of variance (ANOVA) was used to detect between group differences at baseline and after lithium treatment. Means \pm standard error of mean were compared between baseline and post-lithium scans using paired t-tests. The BOLD magnitude after treatment, for each patient, was then subtracted from the BOLD magnitude at baseline and the mean change in BOLD signal magnitude was calculated for each group (depressed and euthymic patients), in each task. A one-way analysis of variance (ANOVA) was carried out to determine between group differences in change in BOLD magnitude due to treatment. Post-hoc analyses using Student's $t$-tests were carried out for each ROI for each group, in each task to determine regional changes in BOLD signal following treatment. Analysis of possible age differences between groups was carried out using twotailed Student's $t$-tests. Statistical significance levels of $\mathrm{p}<$ 0.05 were used throughout.

\section{Results \\ Subjects}

A total of 5 depressed bipolar patients and 5 euthymic bipolar patients took part in the study. Patient characteristics are shown in Table 1 . The mean age of the euthymic patients was $30 \pm 4.8$ of whom 4 were female and of the depressed patients the mean age was $30 \pm 2.1$ of whom 4 were female. There was no statistically significant 
Table I: Patient characteristics. This table shows the age, sex, type of bipolar disorder (BPD), either Type I (I) or Type II (II), as well as the score on the Hamilton Depression scale (HAM-D) and the Young mania rating scale (YMRS).

\begin{tabular}{|c|c|c|c|c|c|c|c|c|}
\hline & Age & Sex & Type of BPD & Ham-D Baseline & Ham-D I4-days & YMRS Baseline & YMRS I4-Days & Medication at time of baseline scan \\
\hline \multicolumn{9}{|c|}{ Euthymic Bipolar Patients } \\
\hline 1 & 26 & $\mathrm{~F}$ & ॥ & 11 & I & 7 & 5 & Sertraline \\
\hline 2 & 22 & $\mathrm{~F}$ & ॥ & 8 & 3 & 4 & 5 & \\
\hline 3 & 27 & $\mathrm{~F}$ & ॥ & 6 & 5 & 9 & 4 & \\
\hline 4 & 26 & $\mathrm{~F}$ & $\|$ & 10 & 7 & 7 & 4 & Venlafaxine \\
\hline 5 & 49 & $M$ & I & 3 & 0 & 3 & 2 & \\
\hline \multicolumn{9}{|c|}{ Depressed Bipolar Patients } \\
\hline 1 & 38 & $\mathrm{~F}$ & 1 & 14 & 9 & 0 & 0 & Quetiapine, venlafaxine, lamotrigine \\
\hline 2 & 27 & $\mathrm{~F}$ & ॥ & 15 & 2 & 7 & 4 & \\
\hline 3 & 32 & $\mathrm{~F}$ & II & 17 & 12 & 2 & i & \\
\hline 4 & 28 & $M$ & 1 & 14 & 17 & I & 1 & Risperidone \\
\hline 5 & 27 & $\mathrm{~F}$ & ॥ & 17 & 15 & 6 & 4 & \\
\hline
\end{tabular}

Table 2: Working Memory Task change in the BOLD signal for each region of interest pre-post lithium The change in each region of interest is shown for the working memory task (mean \pm SEM). There are three treatment groups: healthy controls, euthymic bipolar patients, and depressed bipolar patients. Significant differences (and trends towards significant differences) between patient group and controls are shown as are significant differences (and trends towards significant differences) between euthymic bipolar patients and depressed bipolar patients.

\begin{tabular}{lcc}
\hline \multicolumn{1}{c}{ Region of interest } & Bipolar Euthymic Mean \pm SEM & Bipolar Depressed Mean \pm SEM \\
\hline Cingulate gyrus & $0.03 \pm 0.12$ & $-0.19 \pm 0.12$ \\
Left dorsolateral prefrontal cortex & $0.27 \pm 0.14$ & $0.03 \pm 0.26$ \\
Right dorsolateral prefrontal cortex & $-0.04 \pm 0.05$ & $-0.14 \pm 0.22$ \\
Left inferior parietal gyrus & $-0.15 \pm 0.21$ & $-0.10 \pm 0.11$ \\
Right inferior parietal gyrus & $-0.09 \pm 0.18$ & $-0.13 \pm 0.17$ \\
Left precentral gyrus & $-0.03 \pm 0.29$ & $0.10 \pm 0.10$ \\
Right precentral gyrus & $0.09 \pm 0.14$ & $-0.03 \pm 0.05$ \\
Left superior parietal gyrus & $-0.16 \pm 0.23$ & $-0.08 \pm 0.23$ \\
Right superior parietal gyrus & $-0.12 \pm 0.23$ & $-0.04 \pm 0.11$ \\
Left inferior frontal gyrus & $0.06 \pm 0.31$ & $0.02 \pm 0.20$ \\
Right inferior frontal gyrus & $0.10 \pm 0.10$ & $0.17 \pm 0.15$ \\
Left insula & $-0.03 \pm 0.07$ & $0.18 \pm 0.15$ \\
Right insula & $0.15 \pm 0.11$ & $0.08 \pm 0.17$ \\
Left middle frontal gyrus & $0.25 \pm 0.17$ & $0.36 \pm 0.13$ \\
Right middle frontal gyrus & $0.17 \pm 0.31$ & $-0.10 \pm 0.20$ \\
Left superior frontal gyrus & $0.04 \pm 0.23$ & $-0.28 \pm 0.09$ \\
Right superior frontal gyrus & $0.29 \pm 0.27$ & $0.03 \pm 0.23$ \\
Left inferior occipital gyrus & $0.31 \pm 0.22$ & $0.39 \pm 0.26$ \\
Right inferior occipital gyrus & $0.06 \pm 0.18$ & $0.19 \pm 0.18$
\end{tabular}

difference in age $(\mathrm{t}=0.07, \mathrm{p}=0.94)$. Of the euthymic patients 4 were Bipolar Type II, compared to 3 of the depressed patients. Two of the euthymic patients were concurrently taking antidepressants, while 2 of the depressed patients were taking other psychiatric medication. There was no psychiatric comorbidity in this group. The mean HAM-D score of the euthymic group was $7.6 \pm$ 1.4 at baseline and after lithium the mean score was $3.2 \pm$ 1.3. The mean HAM-D score of the depressed group was $15.4 \pm 0.7$ at baseline and after lithium the mean score was $11.0 \pm 2.6$. This represents an approximate $30 \%$ decrease in HAM-D scores from the initial (mild) scores.

\section{Working memory task performance}

There were no statistically significant differences between these groups on either measure of reaction time at baseline or after treatment (euthymic 529.66 \pm 62.4 and depressed 485.91 \pm 53.0 , at baseline and euthymic 517.84 \pm 36.5 and depressed $537.32 \pm 23.8$ after treatment for the control "arrow" condition; euthymic 907.85 \pm 82.3 and 
Table 3: Word Generation Task change in the BOLD signal for each region of interest pre-post lithium The change in each region of interest is shown for the working memory task (mean \pm SEM). There are three treatment groups: healthy controls, euthymic bipolar patients, and depressed bipolar patients. Significant differences (and trends towards significant differences) between patient group and controls are shown as are significant differences (and trends towards significant differences) between euthymic bipolar patients and depressed bipolar patients.

\begin{tabular}{lcc}
\hline \multicolumn{1}{c}{ Region of interest } & Bipolar & Euthymic Mean \pm SEM \\
\hline Cingulate gyrus & $-0.62 \pm 0.22$ & $-0.13 \pm 0.22$ \\
Left dorsolateral prefrontal cortex & $-0.47 \pm 0.27$ & $0.01 \pm 0.23$ \\
Right dorsolateral prefrontal cortex & $-0.76 \pm 0.40$ & $-0.14 \pm 0.07$ \\
Left inferior parietal gyrus & $-0.65 \pm 0.39$ & $-0.24 \pm 0.33$ \\
Right inferior parietal gyrus & $-0.60 \pm 0.32$ & $0.13 \pm 0.14$ \\
Left precentral gyrus & $-0.75 \pm 0.27$ & $0.68 \pm 0.39 * 1$ \\
Right precentral gyrus & $-0.25 \pm 0.16$ & $-0.02 \pm 0.03$ \\
Left superior parietal gyrus & $-0.87 \pm 0.30$ & $-0.05 \pm 0.47$ \\
Right superior parietal gyrus & $-0.65 \pm 0.21$ & $-0.38 \pm 0.66$ \\
Broca's area & $-0.46 \pm 0.25$ & $0.89 \pm 0.31 * 2$ \\
Left superior temporal gyrus & $-0.94 \pm 0.26$ & $-0.20 \pm 0.22$ \\
Right superior temporal gyrus & $-0.18 \pm 0.05$ & $-0.04 \pm 0.07$ \\
Supplementary motor area & $-0.77 \pm 0.21$ & $0.11 \pm 0.12 * 3$ \\
Thalamus & $0.04 \pm 0.14$ & $-0.04 \pm 0.15$ \\
\hline
\end{tabular}

$* 1 p=0.024$ Euthymic vs Depressed

$* 2 p=0.015$ Euthymic vs Depressed

*3 $p=0.010$ Euthymic vs Depressed

depressed $885.50 \pm 72.7$, at baseline and euthymic 812.86 \pm 25.3 and depressed $908.09 \pm 67.5$ after treatment, for the 5 digit task). There was also no significant difference between the groups on measures of the change in reaction time over after treatment. In the arrow task the change in reaction time was $-11.52 \pm 62.1$ for the euthymic group and $51.4 \pm 34.7$ for the depressed group $(\mathrm{p}=0.46)$. On the 5 digit task the mean change in reaction time for the euthymic group was $-95.8 \pm 88.3$ and $22.6 \pm 11.8(\mathrm{p}=$ 0.31 ).

\section{fMRI results}

In the working memory task over all the regions of interest the mean BOLD signal \pm SEM was $1.02 \pm 0.04$ for the euthymic group, and $1.12 \pm 0.06$ for the depressed group at baseline. After 14 days of lithium the mean signal \pm SEM was $1.08 \pm 0.05$ for the euthymic group, and $1.14 \pm$ 0.07 for the depressed group. Neither of these values varied statistically from baseline ( $p=0.149$ for the euthymic patients and $p=0.56$ for the depressed patients). The mean change in BOLD signal due to treatment was $0.06 \pm$ 0.04 for the euthymic group and $0.02 \pm 0.04$ for the depressed group. These mean changes in BOLD signal were not significantly different between the groups $(\mathrm{F}=$ $0.412, \mathrm{p}=0.522$ ). The results for the BOLD signal change in each region of interest are shown in Table 2.

In the word generation task over all the regions of interest the mean BOLD signal \pm SEM was $1.56 \pm 0.10$ for the euthymic group, and $1.49 \pm 0.10$ for the depressed group at baseline. After 14 days of lithium the mean signal \pm SEM was $1.00 \pm 0.07$ for the euthymic group, and $1.53 \pm$ 0.11 for the depressed group. The euthymic group, but not the depressed group, was statistically different from baseline $(p=0.000$ for the euthymic patients and $p=0.61$ for the depressed patients). The mean change in BOLD signal due to treatment was $-0.57 \pm 0.07$ for the euthymic group and $0.04 \pm 0.08$ for the depressed group $(\mathrm{F}=31.07, \mathrm{p}=$ 0.000). Post-hoc ROI analysis showed that the mean changes in BOLD signal due to treatment were statistically significant in Broca's area, the left pre-central gyrus, and the supplemental motor area (Table 2).

\section{Discussion}

The results from the present study show that following lithium there are differences between patients who are euthymic compared to those who are depressed. The results from the present study suggest that following lithium administration there is alteration in activation in specific brain regions during a word generation task in euthymic, but not depressed, bipolar patients. However, there appears to be no effect in either group in a working memory task. Interestingly, in previous work in healthy volunteers (unpublished information) using the same two cognitive tests we found that 14 days administration of lithium also led to a decrease in mean BOLD levels in the same regions of interest in the word generation task, but had no significant effects on the working memory task. It is possible that one of the reasons that the depressed group didn't show similar changes is that for 
these to be seen the patient must not be depressed. As we only tested these patients after 2 weeks of lithium, at which point they had only had a partial improvement in mood, it is possible that we would have seen changes in this group after a longer period.

Given findings from previous studies that in euthymic patients there are abnormalities of sustained attention, executive functioning, and verbal learning [1,2] it may have been anticipated that lithium may have also affected brain activation during the working memory task. Nonetheless, since lithium may itself have effects on verbal memory $[4,5]$ it is conceivable that these two effects cancelled each other out. Also, one of the limitations of this study was the fact that a more detailed cognitive battery was not performed, and so it is not possible to determine what the cognitive abilities of this group of patients was before and after lithium treatment.

Other limitations of the study are the small size in each group. However, they are well matched on age, sex-distribution, and the number of type I and type II patients which are all factors that could potentially affect the results. Also, both groups each had 3 individuals in whom lithium was the only medication. Thus it is unlikely that these results reflect differences in any of these factors. Nonetheless, future studies should examine larger groups of patients, examine different mood stabilizers in addition to lithium, perhaps examine patients with more severe depression, and follow patients up for a longer period.

Recognizing the possible limitations of this study, one other fMRI study has examined euthymic and depressed bipolar patients [7], although this was on a single occasion. This study suggested there were both traitrelated and state-related changes. Two other fMRI studies have also found differences between controls and euthymic patients $[8,9]$. There also appear to be differences between depressed bipolar patients and controls [21-23].

In conclusion, this is the first study to examine bipolar patients with fMRI before and after lithium administration. It suggests that lithium has effects on brain activation which are task, region, and state-dependent. These findings are in keeping with the current literature, which is clearly somewhat limited at present. More studies are required to answer some of the important outstanding questions.

\section{Acknowledgements}

This work was supported in part by peer-reviewed grants from the Canadian Institutes of Health Research (ClHR) and the Alberta Heritage Foundation for Medical Research (AHFMR).

\section{References}

I. Quraishi S, Frangou S: Neuropsychology of bipolar disorder: a review. J Affect Disord 2002, 72:209-26.

2. Altshuler LL, Ventura J, van Gorp WG, Green MF, Theberge DC, Mintz ]: Neurocognitive function in clinically stable men with bipolar I disorder or schizophrenia and normal control subjects. Biol Psychiatry 2004, 56:560-9.

3. Donaldson S, Goldstein LH, Landau S, Raymont V, Frangou S: The Maudsley Bipolar Disorder Project: the effect of medication, family history, and duration of illness on IQ and memory in bipolar I disorder. J Clin Psychiatry 2003, 64:86-93.

4. Pachet AK, Wisniewski AM: The effects of lithium on cognition: an updated review. Psychopharmacology (Berl) 2003, I 70:225-34. Epub 2003 Sep 19.

5. van Gorp WG, Altshuler L, Theberge DC, Wilkins J, Dixon W: Cognitive impairment in euthymic bipolar patients with and without prior alcohol dependence. A preliminary study. Arch Gen Psychiatry 1998, 55:41-6.

6. Kruger S, Seminowicz D, Goldapple K, Kennedy SH, Mayberg HS: State and trait influences on mood regulation in bipolar disorder: blood flow differences with an acute mood challenge. Biol Psychiatry 2003, 54:I274-83.

7. Blumberg HP, Leung HC, Skudlarski P, Lacadie CM, Fredericks CA, Harris BC, Charney DS, Gore JC, Krystal JH, Peterson BS: A functional magnetic resonance imaging study of bipolar disorder: state- and trait-related dysfunction in ventral prefrontal cortices. Arch Gen Psychiatry 2003, 60:601-9.

8. Strakowski SM, Adler CM, Holland SK, Mills N, DelBello MP: A preliminary FMRI study of sustained attention in euthymic, unmedicated bipolar disorder. Neuropsychopharm 2004, 29: $1734-40$

9. Adler CM, Holland SK, Schmithorst V, Tuchfarber MJ, Strakowski SM: Changes in neuronal activation in patients with bipolar disorder during performance of a working memory task. Bipolar Disord 2004, 6:540-549.

10. Geddes JR, Burgess S, Hawton K, Jamison K, Goodwin GM: Longterm lithium therapy for bipolar disorder: systematic review and meta-analysis of randomized controlled trials. Am J Psychiatry 2004, 161:217-2.

II. American Psychiatric Association: Diagnostic and statistical manual of mental disorders 4th edition. Washington, DC: American Psychiatric Association; 1994.

12. Hamilton M: A rating scale for depression. Neurol Neurosurg Psychiatry 1960, 23:56-62.

13. Young RC, Biggs JT, Ziegler VE, Meyer DA: A rating scale for mania: reliability, validity and sensitivity. Br J Psychiat 1978, 133:429-35.

14. Willson MC, Wilman AH, Bell EC, Asghar SJ, Silverstone PH: Dextroamphetamine causes a change in human neural activity in vivo during cognitive tasks: An fMRI study utilizing BOLD. Biol Psychiatry 2004, 56:284-291.

15. SPM99: Wellcome Department of Cognitive Neurology, University College London.

16. Friston KJ, Worsley K, Frackowiak RSJ, Mazziotta JC, Evans AC: Assessing the significance of focal activation using their spatial extent. Hum Brain Mapp 1994, I:2 10-220.

17. Tzourio-Mazoyer N, Landeau B, Papathanassiou D, Crivello F, Etard O, Delcroix N, Mazoyer B, Joliot M: Automated anatomical labeling of activations in SPM using a macroscopic anatomical parcellation of the MNI MRI single-subject brain. Neuroimage 2002, I 5:273-289.

18. Rorden C, Brett M: Stereotaxic display of brain lesions. Behavioral Neurol 2000, I 2:191-200.

19. Brett M, Anton JL, Valabregue R, Poline JB: Neuroimage 2002, I 6:497. abstract

20. Mulderink TA, Gitelman DR, Musulam MM, Parrish TB: On the use of caffeine as a constrast booster for BOLD fMRI studies. Neuroimage 2002, I 5:37-44.

21. Malhi GS, Lagopoulos J, Ward PB, Kumari V, Mitchell PB, Parker GB, Ivanovski $B$, Sachdev $P$ : Cognitive generation of affect in bipolar depression: An fMRI study. Eur J Neurosci 2004, I 9:74I-54.

22. Caligiuri MP, Brown GG, Meloy MJ, Eberson SC, Kindermann SS, Frank LR, Zorrilla LE, Lohr JB: An fMRI study of affective state and medication on cortical and subcortical brain regions during motor performance in bipolar disorder. Psychiatry Res 2003, I 23: $171-82$. 
23. Caligiuri MP, Brown GG, Meloy MJ, Eyler LT, Kindermann SS, Eberson $S$, Frank LR, Lohr JB: A functional magnetic resonance imaging study of cortical asymmetry in bipolar disorder. Bipolar Disord 2004, 6: 183-96.

Publish with Bio Med Central and every scientist can read your work free of charge

"BioMed Central will be the most significant development for disseminating the results of biomedical research in our lifetime. " Sir Paul Nurse, Cancer Research UK

Your research papers will be:

- available free of charge to the entire biomedical community

- peer reviewed and published immediately upon acceptance

- cited in PubMed and archived on PubMed Central

- yours - you keep the copyright

Submit your manuscript here:

http://www.biomedcentral.com/info/publishing_adv.asp 